\title{
How can the Fourier Transform be Used in Radio Astronomy to Perform Spectral Analysis of Pulsars?
}

\author{
Pranesh Kumar ${ }^{1,2}$ and Arthur Western² \\ ${ }^{1}$ QuartzHigh School, Lancaster, CA, USA \\ ${ }^{2}$ Pioneer Academics, Philadelphia, PA, USA \\ DOI: https://doi.org/10.47611/jsrhs.v10i2.1467
}

\section{ABSTRACT}

The analysis of pulsars is a complicated procedure due to the influence of background radio waves. Special radio telescopes designed to detect pulsar signals have to employ many techniques to reconstruct interstellar signals and determine if they originated from a pulsating radio source. The Discrete Fourier Transform on its own has allowed astronomers to perform basic spectral analysis of potential pulsar signals. However, Radio Frequency Interference (RFI) makes the process of detecting and analyzing pulsars extremely difficult. This has forced astronomers to be creative in identifying and determining the specific characteristics of these unique rotating neutron stars. Astrophysicists have utilized algorithms such as the Fast Fourier Transform (FFT) to predict the spin period and harmonic frequencies of pulsars. However, FFT-based searches cannot be utilized alone because low-frequency pulsar signals go undetected in the presence of background radio noise. Astrophysicists must stack up pulses using the Fast Folding Algorithm (FFA) and utilize the coherent dedispersion technique to improve FFT sensitivity. The following research paper will discuss how the Discrete Fourier Transform is a useful technique for detecting radio signals and determining the pulsar frequency. It will also discuss how dedispersion and the pulsar frequency are critical for predicting multiple characteristics of pulsars and correcting the influence of the Interstellar Medium (ISM).

\section{Introduction}

The discovery of the first pulsar dates back to when Jocelyn Bell was studying radio sources using a radio telescope designed by her advisor, Antony Hewish (Alan, 2006). Both scientists observed radio signals that originated from the same location in the sky and were separated by precisely 1.33 seconds. Bell and Hewish initially ruled out natural sources of the intermittent radio pulses, joking that they came from "little green men." However, after discovering a second pulsating source, the "LGM (Little Green Men) Hypothesis" was abandoned in favor of a new discovery, later deemed the pulsar.

In astrophysics, pulsars are rapidly rotating neutron stars that produce pulses of electromagnetic radiation at their spin rate. The rotating magnetic field serves as the star's generator, accelerating charged particles that travel along the field lines (Ryden, 2003). These particles move relativistically, causing them to produce a large stream of electromagnetic radiation confined to two narrow beams along the pulsar's magnetic poles. Typically, the magnetic poles do not align with the pulsar's spin axis, and the pulses of radiation sweep around like the light of a lighthouse (Cofield, 2016). This phenomenon results in an evenly spaced series of electromagnetic pulses that ground-based telescopes can detect.

Special radio telescopes are capable of determining variation in the successive pulses. However, detecting these signals can be challenging because background radio waves (referred to as noise) swamp weak pulses. One attempt at detecting these precise radio waves involves folding, which is when astronomers stack up many pulses to build a detectable signal. This process is impractical on its own because, for successive pulses to overlap, knowledge of the pulsar's period should be known ahead of time. Folding also needs to be performed on every observation at 
every possible period. Therefore, astronomers use a mathematical function known as the Fourier Transform to detect radio signals and identify essential characteristics of pulsars.

When pulsar signals eventually reach Earth, the low-frequency components are delayed due to interactions with electrons in the interstellar medium (ISM). Despite having a small density, the ISM slightly redshifts radiation detected on Earth. Photons propagating through the ISM are dispersed as a function of group velocity, leading to a frequency-dependent dispersive delay $(\Delta t)$ along the path of the radiation (Bentum, Bonetti, Spallicci, 2016). To determine the actual spin frequency of a pulsar from the data of a dispersed signal, astronomers employ coherent dedispersion. This technique involves the deconvolution of the energy of the emitted radiation with a Fourier Transform function (Bassa \& Hessels \& Pleunis, 2016). Coherent dedispersion involves heavy computation, but it is the dominant technique that utilizes the Discrete Fourier Transform to correct dispersion.

\section{How the Fourier Transform is Involved in Detecting Pulsar Data}

\section{The Discrete Fourier Transform and Nyquist-Shannon Theorem}

The Fourier transform is a reversible, linear mathematical transform used to decompose the graph of a signal from its time domain to the frequency domain. As shown by the definition of the continuous Fourier Transform in Equation 1 (Condon and Ransom, 2016), any integrable function $f(x)$ has a Fourier transform $F(s)$ of the real variable $s$. One unique component of the Fourier transform is that multiplying the variables $x$ and $s$ together will produce a product that is always dimensionless. For example, the Fourier transform of a waveform function $f(t)$ expressed in units of seconds $(s)$ results in a frequency domain signal $F(v)$ written using the units $s^{-1}$, which is equal to $\mathrm{Hz}$.

Equation 1: Definition of the Continuous Fourier Transform

$$
F(s)=\int_{-\infty}^{\infty} f(x) e^{-2 \pi i s x} d x
$$

Practical applications of the Fourier Transform do not utilize the continuous Fourier transform because it involves transforming a time-domain signal of infinite duration. This theoretically requires sampling an endless amount of data. Therefore, astronomers use the Discrete Fourier Transform (DFT) formula shown in Equation 2 (Condon and Ransom, 2016) to convert a signal known at $N$ points to the frequency domain. Graphs of the complex DFT contain a range of frequencies $k$ that begin at - (N/2 - 1) and go to the highest frequency $N / 2$. However, the useful information in a Fourier Transform typically comes from positive frequencies.

Equation 2: Definition of the Discrete Fourier Transform

$$
X_{k}=\sum_{j=0}^{N-1} X_{j} e^{-2 \pi i j k / N}
$$

The range of frequencies in the DFT is determined based on the time interval $t_{s}$ that is used to sample data from a radio telescope. According to the Nyquist-Shannon theorem, or sampling theorem, any continuous function with a known frequency range $(\Delta v)$ of the time domain signal can be reconstructed precisely with a maximum sampling period of $1 /(2 \Delta v)$. Equation 3 (Condon and Ransom, 2016) shows the equation for the Nyquist Frequency, a value describing the maximum observable frequency in a signal's DFT. Knowledge of the Nyquist frequency and sampling period allows astronomers to prevent aliasing and evenly space out acquired data so that it can be utilized in the process of data folding. 
Equation 3: Definition of the Nyquist Frequency

$$
V_{N / 2}=1 /(2 \Delta t)
$$

\section{The Role of the Fast Fourier Transform and Folding in Detecting Pulsar Data}

Digital signal processing (DSP), commonly used in modern radio astronomy, takes advantage of the Fast Fourier Transform (FFT) algorithm to produce a signal's DFT and determine if it originated from a real pulsar (Price, Kocz, Bailes, 2017). The frequency-domain of a pulsar signal contains large "spikes," indicating the fundamental spin frequency and the multiple harmonic frequencies that add a richness to the pulsar signals. Software programs store the power spectrum from these signals in frequency bins, which are equal segments $[f l, f h]$ on the frequency axis containing different signal power values. Figure 1 shows that, even in a pure sine wave sample, there is not a precise peak showing the frequency because of the finite duration of the signal and discrete sampling of the wave.
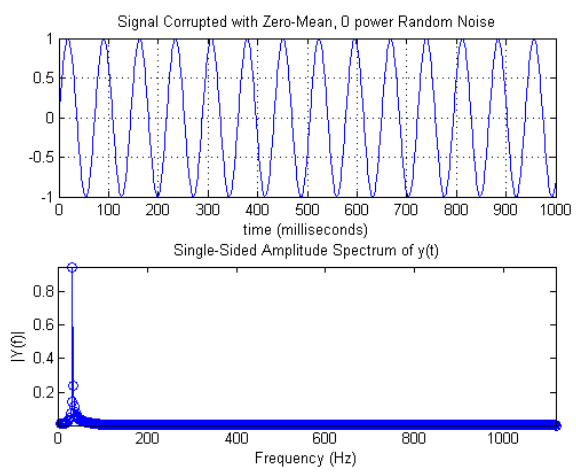

Figure 1. A sample DFT of pure sine wave using frequency bins from DSP Stack Exchange

The Fourier Transform was initially used in blind searches for pulsars to determine the spin period and pulsar frequency from signal data. Astronomers first sampled and transformed "test" signals, such as sine and sawtooth functions of an array of integers, using the sampling period predicted by the Nyquist-Shannon theorem (Nervosa, 2006). Afterward, if the generated power spectrums in the frequency domain were accurate, instruments such as the Ross Xray Timing Explorer (RXTE) would programmatically implement the FFT on signal data to detect pulsar signals. The Discrete Fourier Transform is useful in the search for pulsars due to its sensitive response to periodicity (Ransom, 2002). However, there are many signals from slowly rotating pulsars that go undetected because the "peaks" of pulsar signals in the frequency domain can be challenging to discern in the presence of background radio waves. The following explanations of flux density, system equivalent flux density, and system temperature will help to explain why the FFT search technique is not sensitive enough to detect many long-period radio pulsars.

Flux density describes the rate at which electromagnetic radiation passing through a surface transfers energy. In the case of pulsars, the system temperature $T_{s y s}$ is the noise influencing the radio telescope that can make a single pulse from a pulsar challenging to detect (Breton, 2008). System equivalent flux density SEFD is the flux density equivalent of the system temperature that is proportional to $2 * T_{s y s}$, as shown in Equation 4 (Kramer and Lorimer, 2004). When analyzing system equivalent flux density, the effective area of the peak antenna $A_{e}$ is constant.

Equation 4: Definition of System Equivalent Flux Density

$$
S E F D=\frac{2 k T_{s y s}}{A_{e}} 10^{26} \mathrm{Jy}
$$


The SEFD is a useful measurement for determining the sensitivity of a pulsar search technique because it relates the system temperature to the mean flux density $S_{v, r m s}$. Equation 5, the flux-density based radiometer formula (Parsons, 2012), shows this relationship. $\tau$ represents the integration time in seconds, and $\Delta v$ is the bandwidth in Hz. However, Equation 5's primary importance in this explanation is to identify the proportionality between the $S E F D$ and $S_{v, r m s}$.

Equation 5: Definition of the Mean Flux Density in Relation to the SEFD

$$
S_{v, r m s}=\frac{S E F D}{\sqrt{T \Delta v}}
$$

A lower $S E F D$ value is desirable for detecting periodic pulsar signals because it means that less added noise $T_{s y s}$ is interfering with the radio signal (Bassa \& Hessels \& Pleunis, 2016). Also, according to the flux-density based radiometer formula, search techniques with a lower SEFD should have a small mean flux density $S_{v, \text { rms }}$ and are therefore more sensitive. For long-period pulsars, the mean flux density detected by the FFT can be much larger than what the radiometer equation predicts because of the strong effect of red noise, or noise that primarily impacts low frequencies. One effective way to recover the loss in sensitivity and be able to detect long-period pulsars involves using the timedomain search technique "folding" in conjunction with the FFT data.

The concept of "folding" the time series of pulsar data begins with an understanding of phase and the phase bin number. In a spherical coordinate system for three-dimensional space, as the azimuthal angle (horizontal angle) of a rotating object represents the phase. Instead of using a 3600 method of measuring phase, one rotation counts as one unit. A phase value of 1.5, for example, indicates the pulsar has rotated one and a half times. For a signal with period $P$ where $\Delta t$ represents the time between two consecutive samples, the time series is graphed as $y(k)$ where $k=$ $0,1,2, \ldots, n-1$.

Equation 6: Definition of the phase value corresponding to $\mathrm{y}(\mathrm{k})$

$$
\emptyset_{k}=\frac{k \Delta t}{P}
$$

The phase corresponding to $y(k)$ is represented by $\emptyset_{K}$ and calculated based on the pulse equation used to calculate the pulsar profile shown in Equation 6 (Kulkarni, 2017). All the phase values fall into a range of 0 to 1 because [ $x$ ] is equal to $x$ - fix $(x)$, with fix $(x)$ being the largest integer value smaller than $\mathrm{x}$. For example, if $x$ equals 2.4 , [x] would have a value of 0.4 because $2.4-2.0=0.4$.

Similar to frequency bins, software programs use equally segmented phase bins $\left[\varnothing_{1}, \varnothing_{\mathrm{k}}\right]$ to display the complete folded pulsar profile over $m$ bins (Olney, 2019). Figure 2 shows one example of a folded pulse profile, with the PULSE@Parkes outreach project recording a phase of approximately 0.8. At each phase bin, the frequency in MHz, the time, and the flux are recorded by off-line software packages and then stacked up on top of each other using the folding technique. 


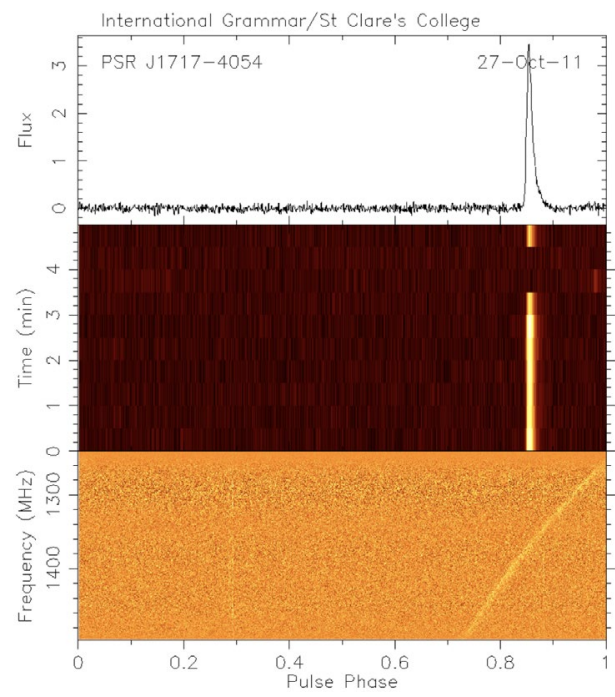

Figure 2. Sample folded pulse profile from the PULSE@Parkes outreach project for PSR J1717-4054

The first step in creating a folded profile is to use the Fast Fourier Transform to determine the spin period of the pulsar. Next, astronomers implement the Fast Folding Algorithm (FFA) to make equally spaced marks in the pulsar data based on the calculated spin period, as shown in the middle section of Figure 3 (Kaspi, Parent, Ransom, 2018). This same algorithm can then add up the signal in each of the folds and "stack up" all the layers together, as shown in Figure 3. Through the FFA algorithm, the pulsar maintains its entire harmonic structure as opposed to the limited number of harmonics in FFT-based searches.

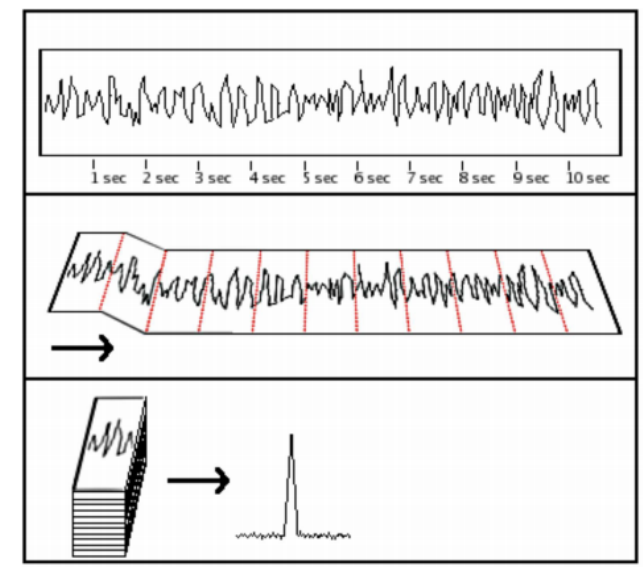

Figure 3. An illustration of the technique of folding from Searching and Identifying Pulsars

In pulsar analysis, the time domain plot in the bottom of Figure $\mathbf{4}$ shows how the signal strength varies through an observation. The darker squares (bins) indicate more significant signal strength and are detected every time a pulsar beam passes the observer, whereas the lighter squares indicate weak signal strength. It is important to note that the spikes in the signal will always occur at the same phase due to the precise periodic timing of the emitted radio signals.

The completed pulse profile, shown above the complete folded profile in Figure 4, is created by adding all the sub-folds together, a process known as integration. Peaks in the pulse profile show that the pulsar is "on," and the 
smaller lines which indicate background noise show when the pulsar is "off" (Noutsos, 2020). Pulsars are present if the complete pulsar profile has a visible pulse.

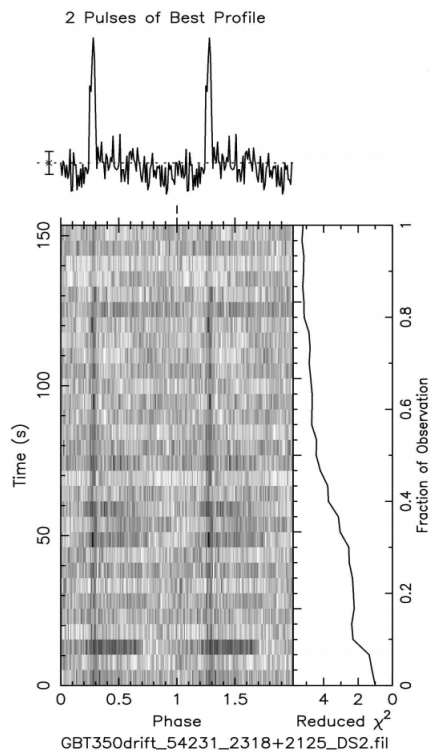

Figure 4. Example Time Series and Pulse Profile from Searching and Identifying Pulsars

\section{Using the Discrete Fourier Transform to Detect Pulsar Features}

\section{Determining the Spin Period using PRESTO software package}

The process of finding and confirming pulsar signals is very computationally demanding, mainly because of the influence of Radio Frequency Interference (RFI). RFI is a hindrance to any search involving radio waves, but it can be especially problematic for pulsar searches (Smyth, 2013). Human-made sources of RFI, such as the alternating current (AC) that supplies our homes, produce intermittent signals that repeat similarly to that of pulsar signals. Therefore, it may appear difficult for radio telescopes to detect the extremely sensitive pulses produced by pulsars and perform spectral analysis based on this data.

One software package that astronomers have created to deal with the difficulty of detecting pulsar signals is PRESTO (Scott Ransom, 2011). The PRESTO package begins by taking the Fourier transform of a time series of signal strength at many different times until it finds a promising candidate. It then measures the observation time using the reference point of an epoch, with Epoch $_{\text {bary }}$ representing the start of the observation. After finding a promising candidate, a program known as prepfold (similar to the Fast Folding Algorithm) is used to fold the time domain data at the pulsar period. The time series and pulse profile are graphed on a prepfold plot and used to determine whether there is significant evidence of a pulsating radio signal coming from a pulsar (Lynch, 2020). 


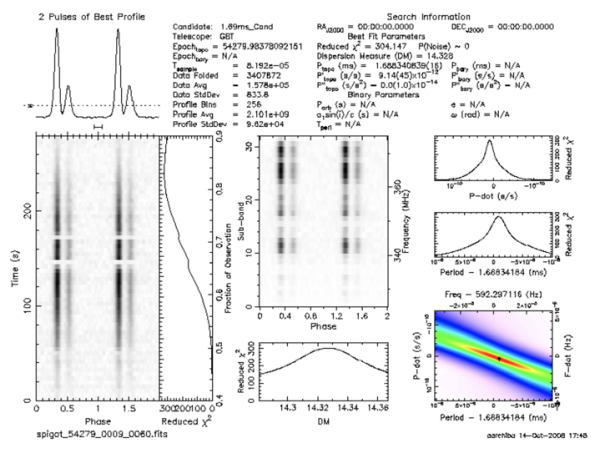

Figure 5: A prepfold plot of the J1023+00 pulsar from the GBT 350-MHz Driftscan survey

As shown from the prepfold plot of the J1023+00 pulsar in Figure 5, many features of a pulsar can be analyzed based on the Fourier transform. This research paper will focus on the data gathered from the J1023+00 pulsar and determine how it can precisely determine the spin period, period derivative, characteristic age, and energy generation rate.

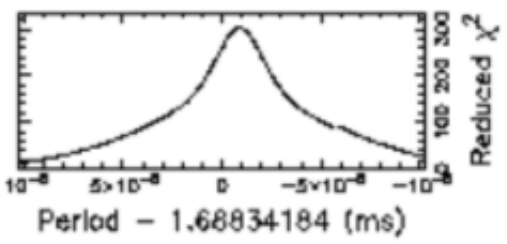

Figure 6: The Spin Period of the J1023+00 Pulsar from the GBT 350-MHz Driftscan survey

The graph in Figure 6 shows the spin period, in milliseconds, of the pulsar on the $\mathrm{x}$-axis and Reduced $\chi 2$ value on the $y$-axis. In statistics, the reduced "chi-squared" $(\chi 2)$ value shows the degree to which a model agrees with some data. Large measurements of the "chi-squared" value typically indicate that there is no significant agreement between the model and data. However, in the scenario of analyzing pulsars, large values are favored because we are comparing a pulsar-less model to data containing no pulsars (Andrae, Schulze-Hartung, Melchior, 2010). Therefore, "peaks" in the graphs of prepfold that show large chi-squared values are indicative of the actual measurements of pulsar characteristics. In the J1023+00 Pulsar, the spin period's value is defined less accurately due to the use of only 256 profile bins when determining features from the data set.

The spin period of the $\mathrm{J} 1023+00$ pulsar is approximately 1.69 milliseconds based on Figure 6 , which means that this pulsar rotates about 592 times a second. J1023+00 is a millisecond pulsar based on this measurement, meaning it likely originated from a globular cluster with an extremely high stellar density.

\section{Using the Pulsar Spin Period to Calculate Additional Features of Pulsars}

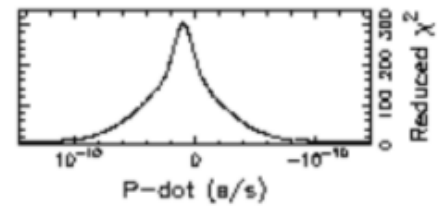

Figure 7: The Period Derivative of the J1023+00 Pulsar from the GBT 350-MHz Driftscan survey 
Figure 7 shows the graph of the P-dot, or first-time derivative indicating how much the period of the J1023+00 pulsar changes over time. The definition of the period derivative shown in Equation 7 (Nervosa, 2006) has a measurement in units of "seconds per second," indicating how many seconds the pulsar's rotation changes after a single second. Pulsars usually behave like most stars, where the spin period increases as a function of time because the rotating neutron star is translating rotational kinetic energy into emitted radiation.

Equation 7: Definition of the Period Derivative

$$
P=\frac{d P}{d t}
$$

A p-dot value that is at approximately zero indicates that the pulsar is isolated because the change in the spin period is not significant enough to be measured by an observation on Earth. In the example of the J1023+00 pulsar, the p-dot value is close enough to zero to where the pulsar is isolated. There is some variability as to the exact period derivative of the pulsar, but the location of the graph's peak indicates that it is approximately $9.14^{*} 10^{\wedge}-12$ seconds per second.

The spin period and period derivative both are useful to calculate an approximate value for the age of a pulsar (characteristic age) because pulsars slow down at a very consistent rate. Equation 8 shows the definition of a pulsar's characteristic age, with $P$ representing the spin period and $d P / d t$ representing the spin period (Nervosa, 2006). This formula approximates the pulsar's age, given the assumption that the pulsar has no magnetic field decay and is in a perfect vacuum. The characteristic age of the J1023+00 pulsar is approximately $9.25^{*} 10^{\wedge} 7$ seconds $(2.93$ calendar years) based on Equation 8, indicating that it is at the beginning of its life-cycle.

Equation 8: Definition of the Characteristic Age

$$
A g e=\frac{P}{2 d P / d t}
$$

The J1023+00 pulsar is classified so far as an isolated, rotationally-powered radio pulsar with a spin period of 1.69 milliseconds and a characteristic age of 2.93 calendar years. Isolated radio pulsars typically have powerful magnetic fields that result in the release of radiation from accelerated charged particles (Condon and Ransom, 2016). The emission of radiation at the magnetic dipoles of rotationally-powered pulsars results in the loss of rotational kinetic energy, which is related to the period and p-dot value. Therefore, astronomers can use the pulsar period and period derivative to calculate the energy generation rate of the $\mathrm{J} 1023+00$ pulsar.

The equation $2 \pi / P$ gives the angular velocity of a rotating object. Plugging this value $\omega$ into the formula for rotational kinetic energy gives the rotational kinetic energy as a function of the pulsar period, shown in Equation 9 (Lyne and Graham-Smith, 2012).

Equation 9: Definition of Angular Velocity for a Rotating Object

$$
K=\frac{1}{2} I \omega^{2}=\frac{2 \pi^{2} I}{P^{2}}
$$

The derivative of the rotational kinetic energy relates directly to the rate at which the kinetic energy is changing, as shown in Equation 10 (Lyne and Graham-Smith, 2012). Given that lost rotational kinetic energy is transferred to the emission of radiation at the magnetic dipoles, the energy generation rate $d E / d t$ of a rotationally-powered pulsar is $-(d K / d t)$. 
Equation 10: Definition of the Derivative of Kinetic Energy

$$
\frac{d K}{d t}=-\frac{4 \pi^{2} I}{P^{3}} \frac{d P}{d t}
$$

Assuming that the $\mathrm{J} 1023+00$ pulsar is a uniform sphere, the moment of inertia, $I$, has an approximate value of $2 / 5 *$ $M * R^{\wedge} 2 . M$ represents the mass of the pulsar, while $R$ represents the pulsar's radius. Neutron stars, in general, have an estimated radius of 10 kilometers and a mass of about 1.4 solar masses due to the precise conditions that are necessary for these celestial objects to exist (Lorimer, 2008). Plugging all of these values into Equation 10 and taking the negative value of $d K / d t$ gives an energy generation rate $d E / d t$ of $2.11^{*} 10^{\wedge} 31$ joules/second. This value shows that the $\mathrm{J} 1023+00$ pulsar releases around $2.11^{*} 10^{\wedge} 31$ joules of energy every second due to charged particles flung from the surface of the star.

The release of energy from pulsars, known as synchrotron radiation, should not be confused with the energy from the much smaller pulses we detect on Earth. These pulses are the result of the individual charged particles emitting beams of radiation along their direction of motion.

\section{Detecting Impact of ISM on Pulse Propagation Through Coherent Dedispersion}

\section{Interstellar Propagation and Dispersion}

Pulsars are classified as steep-spectrum radio sources, meaning that they produce compact radio signals with a peak frequency at only a few hundred MHz. In the same way that optical light undergoes dispersion when it passes through water, intermittent radio waves produced by pulsars are significantly dispersed as they pass through the interstellar medium. The interstellar medium (ISM), although partly consisting of cold clouds of neutral hydrogen atoms, contains diffuse regions with ionized hydrogen and free electrons (Smith, 1999). Pulsar signals that pass through these electrons experience a time delay $t$ relative to their radio frequency, as shown in Equation 11.

Equation 11: Definition of time delay using the dispersion measure value.

$$
t=\frac{D M}{2.42 \times 10^{-16} v^{2}}(s)
$$

The value $D M$ in this equation represents the dispersion measure, which, by definition, is the "integrated column density of free electrons between an observer and a pulsar." Another way to think of the dispersion measure is the number of free electrons between the observer and pulsar per unit area (Lyon, 2016). Energetic radio waves with a higher frequency $\nu$ lose very minimal speed when they travel past charged particles in the interstellar medium. However, low-frequency signals in the presence of charged particles slow down more significantly due to refraction. This phenomenon leads to a pulsar signal of bandwidth $B$ to have the low and high ends of the bandwidth to be received at different times, as shown in Figure 8 (McKee, 2018). The x-axis represents the time for one "pulse phase" (period), and the $y$-axis represents the radio frequency. A clear pattern in Figure 8 depicts how radio telescopes detect the low frequency components of the signal after the high frequencies. 


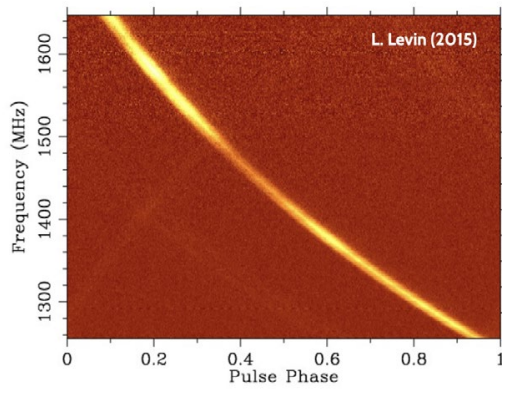

Figure 8: A Graph Depicting Dispersion as Being Influenced by Frequency from the Max Planck Institute for Radio Astronomy

The delay resulting from the interaction of radio waves with charged particles causes the profile to undergo dispersive "smearing." Dispersive smearing results in the spike of the pulsed signal being broadened at higher pulse phases, as shown in the bottom section of Figure 9 (Lorimer, 2008). When radio telescopes first observe pulsar signals, scatterbroadening at lower frequencies decreases the observational sensitivity and timing precision.

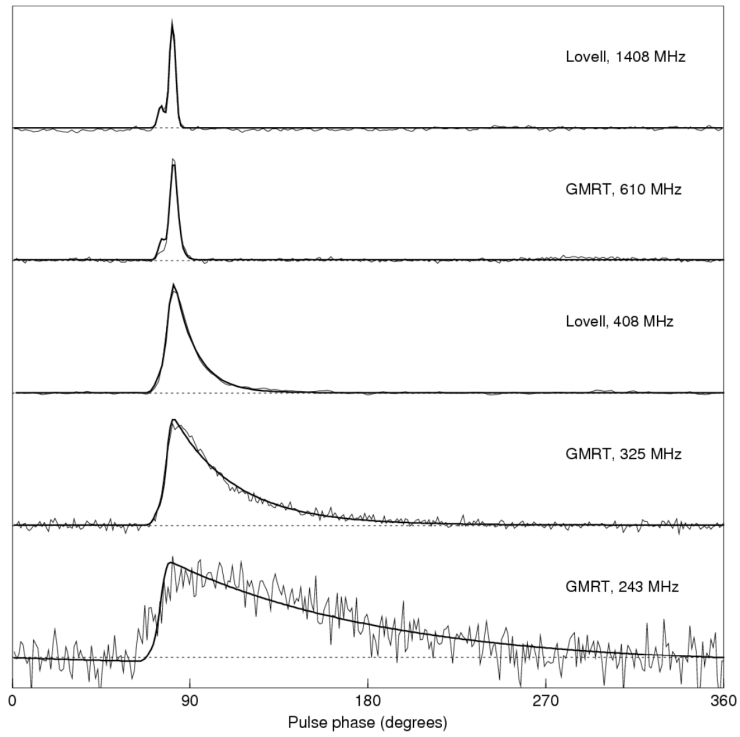

Figure 9: Pulse Broadening Caused by Scattering from the Handbook of Pulsar Astronomy

\section{Using Coherent Dedispersion to Remove Dispersive Effects of ISM}

For pulsar searches, the dispersion measure (DM) is a search parameter that specifies the amount of time by which the interstellar medium smears signals $t_{\text {smear }}$. Astronomers specifically aim to narrow the bandwidth $B$ to decrease the $t_{\text {smear }}$ value (Smyth, 2013). Instruments have implemented ways of observing many small signal channels and combining all the signals to create a larger bandwidth, intending to limit the impacts of a large bandwidth on dispersive smearing.

Adding the frequencies together with the predicted dispersive delay was initially used in incoherent dedispersion to reconstruct an original pulsar signal (Barsdell, Bailes, Barnes, 2012). Astronomers nowadays use software 
to perform coherent dedispersion, a process modeling the effect of the ISM on radio propagation as a linear filtering operation. The ISM filter can be modeled as a transfer function $H(v)$ and multiplied with the Fourier Transform of the incoming signal $F(v)$ to produce the Fourier Transform of the filtered signal $G(v)$.

The transfer function $H(v)$ is depicted using the notation $H\left(v+v_{0}\right)$, where $v_{0}$ is the central observing frequency. The bandwidth of the signal is bounded by $\pm v . H\left(v+v_{0}\right)$ is calculated using the transfer function formula shown in Equation 12 (Mckee, 2018), written in terms of the wavenumber $k(v)$ and the distance from the pulsar $d$.

Equation 12: Transfer function formula in terms of the wavenumber and fundamental frequency

$$
H\left(f_{0}+f\right)=\exp \left[-i k\left(f_{0}+f\right) d\right]
$$

Astronomers have performed further calculations to write the wavenumber $k(v)$ in terms of the dispersion measure $D M$ and bandwidth frequencies, resulting in the modified transfer function shown in Equation 13 (Stairs, 2002). By convolving the discrete Fourier Transform of the observed signal with the inverse transfer function $H(v+v 0)$, the Fourier Transform of the filtered signal $G(v)$ will be almost perfectly aligned. Coherent dedispersion requires heavy computation by software programs, but it is still a valuable technique to determine the accurate pulsar profile of a high sensitivity signal.

Equation 13: Modified transfer function formula which is convolved with the DFT

$$
H\left(v_{0}+v_{1}\right)=\exp \left[2 \pi i \frac{D M}{2.41 \times 10^{-10}} \frac{v_{1}^{2}}{v_{0}^{2}\left(v_{0}+v_{1}\right)}\right]
$$

\section{Conclusion}

The Discrete Fourier Transform is a valuable mathematical technique that has allowed astronomers to determine innovative solutions for performing spectral analysis of pulsars. Radio Frequency Interference (RFI) makes the process of detecting and analyzing pulsar signals through blind searches very difficult. This combined with the relatively few numbers of pulsars detected, has forced astronomers to be creative in the techniques they use to determine the specific characteristics of these unique rotating neutron stars.

By sampling the time domain data of a pulsating signal using the Nyquist-Shannon theorem, algorithms such as the Fast Fourier Transform (FFT) have been used to predict the spin period and harmonic frequencies. However, it was quickly realized that astronomers could not use FFT-based searches alone because low-frequency pulsar signals can go undetected in the presence of background noise. To improve the sensitivity, astronomers have used folding to stack up many pulses at the pulse period. Folding is impractical on its own, but the computation needed to perform this technique has been made substantially easier thanks to the Fast Folding Algorithm.

Though coherent dedispersion is mathematically precise it comes at the cost of being computationally demanding. Astronomers still utilize this technique because of the noticeable improvement in sensitivity compared to incoherent dedispersion. Coherent dedispersion is critical because it utilizes the Discrete Fourier Transform to accurately display pulsar signals in the time domain.

\section{Acknowledgments}

I would like to personally thank Professor Arthur Western and the Pioneer Academics Research Program team for consistently supporting me in the completion of this challenging project. 


\section{References}

A. Chodos. (2006). This Month in Physics History: February 1968: The Discovery of Pulsars Announced. American Physical Society, Volume 15, Number 2. Retrieved from https://www.aps.org/publications/apsnews/200602/his$\underline{\text { tory.cfm }}$

A. Lyne, F. Graham-Smith. (2012). Pulsar Astronomy 4th Edition. Cambridge Astrophysics, Series Number 48.

A. Nervosa. (2006). Detecting Pulsars. Retrieved from http://astronomyonline.org/Stars/Pulsars.asp

A. Noutsos, G. Desvignes, M. Kramer, N. Wex, P. C.C. Freire, et al. (2020). Understanding and improving the timing of PSR J0737-3039B. Astronomy \& Astrophysics 643, A143. DOI:https://doi.org/10.1051/0004$\underline{6361 / 202038566}$

A. Parsons. (2012). Radiometer Equation Applied to Telescopes. AstroBaki. Retrieved from https://casper.ssl.berkeley.edu/astrobaki/index.php/Radiometer Equation Applied to Telescopes

B.R. Barsdell, M. Bailes, D.G. Barnes, \& C.J. Fluke. (2012). Accelerating Incoherent Dedispersion. Monthly Notices of the Royal Astronomical Society, Volume 422, Issue 1. DOI:https://doi.org/10.1111/j.13652966.2012.20622.x

B.R. Ryden. (2003). Astronomy 162: Pulsars. Retrieved from http://www.astronomy.ohio-state.edu/ ryden/ast162 5/notes2

C. Cofield. (2016). What Are Pulsars?. Retrieved from https://www.space.com/32661-pulsars.html

C.G. Bassa, Z. Pleunis, J.W.T Hessels. (2016). Enabling Pulsar and Fast Transient Searches using Coherent Dedispersion. Instrumentation and Methods for Astrophysics. DOI:https://doi.org/10.1016/J.ASCOM.2017.01.004

D.C. Prince, J. Kocz, M. Bailes, L.J. Greenhill. (2017). Introduction to the Special Issue on Digital Signal Processing in Radio Astronomy. Journal of Astronomical Instrumentation.

DOI:https://doi.org/10.1142/S2251171716020025

D.R. Lorimer \& M. Kramer. (2004). Handbook of Pulsar Astronomy. Cambridge University Press, Vol. 4.

D. R. Lorimer. (2008). Binary and Millisecond Pulsars. Living Reviews in Relativity.

DOI:https://doi.org/10.12942//rr-2008-8

D. Smyth. (2013). Handbook on Radio Astronomy Third Edition. Radiocommunication Bureau Edition of 2013.

E. Parent, V. M. Kaspi, S. M. Ransom, M. Krasteva, et al. (2018). The implementation of a Fast-Folding pipeline for long-period pulsar searching in the PALFA survey. High Energy Astrophysical Phenomena.

DOI:https://doi.org/10.3847/1538-4357/aac5f0

G. Hobbs, R. Hollow, J.K. Khoo. (2009). The PULSE@Parkes Project: a New Observing Technique for Long-Term Pulsar Monitoring. Publications of the Astronomical Society of Australia 26(4).

DOI:https://doi.org/10.1071/AS09021 
G. Smith. (1999). Gene Smith's Astronomy Tutorial: The Interstellar Medium. University of California, San Diego Center for Astrophysics \& Space Sciences. Retrieved from https://casswww.ucsd.edu/archive/public/tutorial/ISM.html

I.H. Stairs. (2002). Pulsar Observations II. -- Coherent Dedispersion Polarimetry, and Timing. Single-Dish Radio Astronomy: Techniques and Applications, Volume 278. DOI: Retrieved from http://adsabs.harvard.edu/pdf/2002ASPC..278..251S

J. Boyles. (2009). GBT 350-MHz Driftscan Survey Processing. Retrieved from https://www.http://astro.phys.wvu.edu/GBTdrift350/

J. Condon \& S. Ransom. (2016). Essential Radio Astronomy. Princeton Series in Modern Observational Astronomy.

J. McKee. (2018). Introduction to Pulsar Timing and Tempo2. Max Planck Institute for Radio Astronomy IPTA Student Week. Retrieved from http://ipta.phys.wvu.edu/files/student-week-2018/pulsar timing lecture.pdf

J. McKee. (2018). The Interstellar Medium. Max Planck Institute for Radio Astronomy IPTA Student Week. Retrieved from http://ipta.phys.wvu.edu/files/student-week-2018/ism lecture.pdf

M.J. Bentum, L. Bonetti, A. Spallicci. (2016). Dispersion by pulsars, magnetars, fast radio bursts and massive electromagnetism at very low radio frequencies. Faculteit van Elektrotechniek, Wiskunde en Informatica, Telecommunication Engineering. DOI:https://doi.org/10.1016/j.asr.2016.10.018

R. Andrae, T. Schulze-Hartung, P. Melchior. (2010). Do and don'ts of reduced chi-squared. Institut für Theoretische Astrophysik, ZAH, Albert-Ueberle-Str. 2. Retrieved from https://www.semanticscholar.org/paper/Dos-anddon\%27ts-of-reduced-chi-squared-Andrae-Schulze-Hartung/353308ea0f2e5be88f397cbd5c0528bc0069208b

R. J. Lyon. (2016). Why are Pulsars Hard to Find?. Submitted to the University of Manchester for the Degree of Doctor of Philosophy in the Faculty of Engineering and Physical Sciences. Retrieved from http://www.scienceguyrob.com/wp-content/uploads/2016/12/WhyArePulsarsHardToFind Lyon 2016.pdf

R.P. Breton. (2008). Radio Pulsars in Binary Systems. Department of Physics McGill University. Retrieved from https://https://arxiv.org/abs/0907.2623

R.S. Lynch. (2020). Searching for and Identifying Pulsars. Department of Physics, McGill University. Retrieved from http://pulsarsearchcollaboratory.com/wp-content/uploads/2016/01/PSC search guide.pdf

S. Juteux, R. Ramachandran, B.W. Stappers. et al. (2002). Searching for Pulsars in Close Circular Binary Systems. Astronomy \& Astrophysics. DOI:https://doi.org/10.1051/0004-6361:20020052

S. Olney. (2019). A Collection of Information About Amateur Pulsar Detection. Neutron Star Group. Retrieved from http://www.neutronstar.joataman.net/

S.R. Kulkarni. (2017). Folding a Time Series. Retrieved from https://sites.astro.caltech.edu/ srk/Ay3/Folding/Folding.pdf

Z. Pleunis, C. G. Bassa, J. W. T. Hessels, V. I. Kondratiev, et al. (2017). A Millisecond Pulsar Discovery in a Survey of Unidentified Fermi $\gamma$-Ray Sources with LOFAR. The Astrophysical Journal Letters, 846:L19 (7pp). DOI:https://doi.org/10.3847/2041-8213/aa83ff 